\section{Cahiers de Narratologie}

Analyse et théorie narratives

22 | 2012

Voix off et narration cinématographique. Second volet

\title{
Voix over et peur au cinéma
}

\section{Martin Barnier}

\section{OpenEdition}

Journals

Édition électronique

URL : http://journals.openedition.org/narratologie/6536

DOI : $10.4000 /$ narratologie. 6536

ISSN : $1765-307 X$

Éditeur

LIRCES

\section{Référence électronique}

Martin Barnier, « Voix over et peur au cinéma », Cahiers de Narratologie [En ligne], 22 | 2012, mis en ligne le 20 juillet 2012, consulté le 02 mai 2019. URL : http://journals.openedition.org/ narratologie/6536 ; DOI : 10.4000/narratologie.6536

Ce document a été généré automatiquement le 2 mai 2019.

Article L.111-1 du Code de la propriété intellectuelle. 


\title{
Voix over et peur au cinéma
}

\author{
Martin Barnier
}

1 La voix humaine peut-elle faire peur? On connait de nombreuses voix de narration qui permettent de voir des documentaires animaliers dans une atmosphère calme et détendue. Les voix d'André Dussolier ou de Pierre Arditi, avec leur grain chaud, leur timbre agréable et leur ton posé donnent une idée rassurante de la vie dans la nature, même lors d'événements dramatiques. Placées sur les images de la vie sauvage, elles sont là pour donner des informations scientifiques, des commentaires amusés et créer une complicité avec le spectateur. Ces voix sont masculines la plupart du temps ${ }^{1}$. Pour désigner ces voix enregistrées dans un studio et posées sur les images, nous utilisons l'expression «voix over». Ce terme a été proposé par Jean Chateauvert ${ }^{2}$ et les théoriciennes américaines comme Sarah Kozloff ${ }^{3}$. Puis, le Suisse Alain Boillat a repris ce terme et développé une théorie . Utiliser " over » évite les confusions avec le mot « off » qui désigne le hors-champ sur les plateaux de cinéma de la plupart des pays du monde.

2 Cette voix over, qui nous rassure dans les documentaires, ou les reportages, peut-elle aussi être effrayante? Quand la voix over est utilisée dans la fiction, elle devient voix de narrateur, nous dit Châteauvert. Le désancrage de la voix par ce procédé, selon l'expression d'Alain Boillat, peut-il expliquer l'utilisation des voix over dans le but d'effrayer? Avec des exemples différents, pris dans l'ordre chronologique, pour la plupart, nous essaierons de déterminer l'évolution de cette voix over d'effroi. Les analyses de séquences nous permettront de comprendre comment la voix de narration, posée sur des images, et qui semblait garantir calme et tranquillité à l'auditeur, peut devenir le plus sûr moyen de créer la peur chez le spectateur.

Les premières voix, accompagnant des images en mouvement, sont celles des commentateurs de vues (lanternes magiques puis films). Après la généralisation du parlant, dans les années 1930 et 1940, la voix over accompagne des films d'horreur. Elle reflète des terreurs dues à l'introspection d'un personnage. Dans les années 1960 et 1970, des voix over de films " giallo ", de science-fiction ou de films gore nous donnent d'autres exemples permettant de comprendre l'évolution de cette forme cinématographique. On 
pourra se demander s'il y a des éléments de continuité entre le bonisseur-conférencier et les voix over plus récentes.

\section{L'explicateur de vues peut-il faire peur?}

4 Le «bonimenteur », qui préférait se faire appeler « conférencier ${ }^{5}$ », est un personnage important dans les salles projetant des films avant 1914. La tradition d'expliquer les «vues» (terme le plus souvent utilisé jusque vers 1910, même s'il s'agissait de films) remonte aux ombres chinoises et aux lanternes magiques. Les historiens de ce qu'André Gaudreault nomme la "cinématographie attraction», insistent aujourd'hui sur la continuité du spectacle de projection de films avec les ombres et les lanternes ${ }^{6}$. Lors des fantasmagories présentées par Robertson entre les années 1790 et 1836, les éléments sonores utilisés ont pour but d'effrayer le spectateur. Quelques paroles peuvent se mêler aux bruits d'orage, de foudre, etc. Dans ce spectacle, «Diogène, sa lanterne à la main, cherche un homme et pour le trouver traverse, pour ainsi dire, les rangs, et cause impoliment aux dames une frayeur dont chacun se divertit ${ }^{7} »$. En dehors des fantasmagories de Robertson, qui furent rapidement imitées, les voix des lanternistes peuvent, tout en expliquant des vues fixes avec calme et pédagogie, vouloir effrayer les auditeurs en faisant croire à l'apparition de fantômes. Ces spectacles existent encore lorsque le cinématographe se développe.

5 Nous n'avons presque aucun enregistrement d'explicateur de film ${ }^{8}$, mais les traces trouvées dans les journaux permettent des hypothèses sur le contenu de certains commentaires. Une salle spécialisée dans la projection de films, avenue de la Gare à Nice ${ }^{9}$, annonce un programme exceptionnel dans les journaux, en 1907 :

Vendredi prochain, M. Schneider donnera une représentation au profit des familles victimes de la catastrophe du «Berlin » à Hœck Van Holland. Le Royal Vüo avait envoyé son représentant de Rotterdam sur les lieux du sinistre pour cinématographier toutes les phases de ce drame de mer :

$1^{\circ}$ L'épave du "Berlin »; les flots disloquant le poids de cette masse [sic] enfin toutes les péripéties du sauvetage, lorsque le navire sombre après une lutte désespérée, engloutissant ceux qu'il portait.

Voilà une vue qui est faite pour déplacer les plus blasés et qui est de circonstance ${ }^{10}$.

Ce texte nous donne une idée des commentaires donnés dans la salle. Il s'agit bien d'effrayer «les plus blasés» avec le spectacle de la mort "en direct» ou presque puisqu'on voit l'épave sombrer « engloutissant ceux qu'elle portait ». On imagine les cris dans le public et le «bonimenteur» surenchérissant sur les images des différents moments de la catastrophe. Ce sont les images qui doivent déclancher l'effroi, d'autant plus qu'il ne s'agit pas d'une fiction, mais le texte du journal indique la façon dont le commentaire devait se faire, accentuant l'horreur du fait-divers.

7 Le commentateur de vues peut aussi encourager la peur chez les spectateurs qui viennent voir une fiction prévue pour effrayer. Un tract publicitaire, rédigé par Louis Feuillade, nous indique sur quel ton l'explicateur de films peut créer une ambiance effrayante pendant la diffusion des Vampires, en novembre 1915 :

Qui sont-ils ? Où sont-ils?

Au seuil de ce mystère

Si tu sens défaillir ton cœur, trembler ta main,

Jette au loin ce papier et passe ton chemin,

Sinon ouvre ce pli... 
La réponse est derrière.

Des nuits sans lune, ils sont les rois,

Les ténèbres sont leur empire.

Portant la Mort, semant l'Effroi,

Voici le vol noir des Vampires

Aux grandes ailes de velours

Non pas vers le Mal...

Mais vers le Pire !11

8 Il s'agit donc de mettre en condition le spectateur pour qu'il soit effrayé par le film réalisé par Feuillade. Le matériel publicitaire et les catalogues envoyés par les distributeurs des films pouvaient inspirer les voix «over» des conférenciers dans les salles de la Belle Époque.

Avec la généralisation du parlant ${ }^{12}$, au début des années 1930, la voix over fit partie des procédés utilisés pour créer une ambiance morbide lorsque le "genre horreur » prit une place de choix dans la production américaine.

\section{Les voix d'horreur des années 1930-1940}

Un des grands succès de la Warner, la première compagnie à produire des longs métrages "100\% parlant» (selon l'expression de l'époque), en 1928, se nomme The Terror (Roy Del Ruth). Sorti le 8 septembre 1928 , le film rapporta plus de $1.220 .000 \$$. Ce fut la cinquième meilleure recette des films sonores et parlants sortis en 1927 et 1928 aux États-Unis ${ }^{13}$. Ce film comprend un générique parlé que récite un personnage masqué (presque over ${ }^{14}$ ). La parole, pour ce deuxième film «100\% parlant » produit par la Warner, sert donc déjà à générer de l'angoisse. Pour créer une atmosphère étrange, un personnage, prévenant le public $d u$ «choc» que représente le film, le présente dans le pré-générique de Frankenstein (James Whale) en 1931. Il semble donc qu'un médiateur entre le public et le film d'horreur qui va se dérouler, soit nécessaire au début du parlant ${ }^{15}$.

11 Une véritable voix over de générique s'entend au début du Mystère de la chambre jaune, dans la version réalisée par Marcel L'Herbier en 1930 (sortie en France en février 1931). Le récitant, qui n'est pas un des personnages de l'histoire, prend un ton lugubre. Il ne fait pourtant que donner la liste des techniciens qui apparaissent à l'image en même temps qu'il cite leur nom. Les noms des chefs opérateurs, ingénieurs du son, monteuses, ont droit à une dramatisation accentuée par un vibrato dans la voix. Les images de l'équipe technique, aussi bien que celles des acteurs, dans un noir et blanc expressionniste, laissent peu d'éléments éclairés, avec des angles de prise de vue en plongées ou contreplongées. La voix du récitant, avec ces images, plonge le spectateur dans un climat angoissant, alors même que le film n'est pas commencé. Le titre apparaît sur un bruit de vent, dans des lettres de feu. C'est le ton utilisé en voix over qui crée de la peur, ou, dans le cas présent, qui prépare l'auditoire à la peur.

12 À l'opposé d'un ton grandiloquent, solennel ou pompeux, comme dans Le Mystère de la chambre jaune, on trouve des voix over d'une grande douceur... qui n'en sont pas moins effrayantes. Juste après le générique, Rebecca, d'Hitchcock (1940), s'ouvre sur la description d'un rêve. Une voix féminine décrit des images oniriques. Elle explique qu'il s'agit de souvenirs. Le film débute donc par une sorte de flash-back. Certains critiques ont trouvé que l'utilisation de la voix over d'un personnage était une façon habile de plonger immédiatement le spectateur dans une atmosphère nostalgique ${ }^{16}$. Dans Rebecca, la voix 
over disparait très vite. Hitchcock va au-delà de la nostalgie : cette voix est douce, tranquille, apaisante. Pourtant, elle nous fait avancer dans un paysage brumeux, découvrir des ruines d'un château brûlé, dans un lieu très inquiétant. Kozloff note que l'image correspond au ton et aux mots de la voix over : la voix guide l'image ${ }^{17}$. Mais nous remarquons surtout un décalage entre la voix (trop) douce et les images lugubres. Le contraste ainsi créé sert à insuffler la peur. Un arbre effrayant, au premier plan, masque en partie le château sombre et détruit, à l'arrière plan, quand la narratrice explique que la lumière de la lune peut jouer des tours et qu'elle imagine qu'il y a encore des pièces éclairées. Après cette description intrigante, les mots « les jours les plus étranges de ma vie, qui commencèrent dans le sud de la France ", permettent à l'histoire de vraiment commencer. On se rend alors compte que cette voix de narration est celle de Joan Fontaine, qui apparaît à l'image, dans le sud de la France. La voix over a guidé le spectateur à travers une grille sur un chemin tortueux, dans le brouillard. Elle a obligé le public à s'interroger devant une ruine lugubre. Même très douce, cette voix ne pourraitelle pas être celle d'un fantôme? Une morte raconterait-elle en flash-back les circonstances d'une fin de vie tragique? Ce sera le cas en ouverture de Sunset boulevard (Billy Wilder, 1950), film tourné dix ans plus tard, qui montre le scénariste déchu, joué par William Holden, noyé dans une piscine. Le calme de la voix over en ouverture de Rebecca laisse la porte ouverte à plusieurs interprétations : une morte qui parle, un simple rêve, un souvenir mélangeant angoisse et bonheur. L'image noire et le lent flottement de la caméra fantomatique contrastent avec l'aspect apaisant de la voix, laissant un doute au spectateur, une place pour l'effroi, qui envahira plusieurs séquences du film.

Une autre production américaine du début des années 1940 propose une voix féminine over qui ne manque pas de troubler l'auditeur. Dans Vaudou (I Walked with a Zombie), réalisé par Jacques Tourneur en 1943, Frances Dee interprète une infirmière canadienne (Betsy Connell) qui arrive à Saint-Sebastian, dans une île des Caraïbes, pour soigner une étrange malade, dans une riche famille de planteurs. La voix over est calme, douce, apaisante. Pourtant, ce qu'elle raconte est effrayant. À la fin du générique, Betsy Connell explique «j'ai marché avec un zombie » et l'on entend son amusement, comme dans un rire étouffé. Se moque-t-elle du spectateur qui doit être effrayé par l'image d'un zombie? Elle précise qu'elle savait "que les zombies sont des créatures étranges et effrayantes ", mais elle le dit avec un détachement complet. Quand l'infirmière arrive devant la villa, les bruitages d'oiseaux s'arrêtent brusquement pour laisser place de nouveau à la voix over accompagnée d'une musique lente, étrange et répétitive. On distingue les différentes parties du jardin et surtout un Saint-Sébastien planté de flèches. Cette figure de proue, qui était à l'avant d'un navire transportant des esclaves africains, a été transformée en fontaine, mais d'aspect sinistre et évoquant le passé esclavagiste de la famille. Sur ces images, la voix over toujours détachée et neutre, prévient le spectateur de l'effroi qui adviendra. Elle explique par exemple: "J'allais entendre une étrange confession que seule la folie explique ».

Cette voix over semble anempathique, comme le dit Michel Chion de la musique qui est en opposition avec l'ambiance d'une séquence ${ }^{18}$. Pour l'auditeur, le film devient encore plus effrayant. La froide distance imposée par la voix de l'infirmière, alors que les images, la musique, et le contenu même des paroles, ne sont qu'horreur et folie, rend l'ensemble glaçant.

15 Une autre voix over des années 1940 peut effrayer le spectateur, mais cette fois de façon plus directe. Il s'agit d'un flash-back sonore, lorsque le personnage de Paula Alquist, joué 
par Ingrid Bergman, se rappelle les phrases prononcées par son horrible mari (Charles Boyer), dans Hantise (Gaslight). Dans ce remake de 1944 d'un film anglais de 1940, George Cukor a placé une voix over qui répète " Ta mère est morte folle, ta mère est morte folle " sur l'image d'Ingrid Bergman tentant de se concentrer sur la lecture (à haute voix) d'un livre. La phrase qui résonne en boucle provient de l'imagination et de la mémoire de la jeune femme. C'est un son interne subjectif ${ }^{19}$. Cette injonction en voix over pousse le personnage vers la folie. Le mari prétend qu'elle est la fille d'une malade mentale. L'esprit de la jeune femme, sous la coupe d'un manipulateur, vacille, et l'on entend deux voix s'opposer : la sienne et celle de son époux qui résonne dans sa tête. Cette voix mentale est effrayante pour le personnage comme pour le spectateur.

Les années 1940 sont celles d'une véritable passion de la production hollywoodienne pour la psychanalyse. La voix de l'inconscient, ou, plus souvent, de la conscience, se mêle, en over, aux sons des films. L'un des spécialistes de ce type de voix mentale est Fritz Lang. En 1945, dans Scarlet Street (La Rue rouge), son remake de La Chienne de Renoir, Lang fait résonner la voix de la culpabilité dans la tête du comptable joué par Edward G. Robinson. À la toute fin du film, alors que Christopher Cross se réfugie dans une chambre d'hôtel, il entend deux voix. Kitty, sa maîtresse, lui rappelle qu'il la assassinée. Johnny, le souteneur de cette dernière, dénonce aussi Christopher (c'est à cause de lui que Johnny est passé sur la chaise électrique). Les deux voix sont susurrées : elles sifflent aux oreilles de Chris, mais elles envahissent l'espace. "You killed me, Chris " répètent les voix de Kitty et Johnny, se chevauchant, interrompues par des rires. Les murmures soufflés des deux personnages sont internes subjectifs. Ils parasitent l'esprit défaillant du comptable meurtrier. La «voix de la conscience » revient torturer celui qui croyait échapper à la justice. Il s'agit d'une belle trouvaille, qui contient des aspects effrayants, obsédants. Lang avait déjà procédé ainsi quand la voix du Docteur Mabuse s'infiltrait en sifflant dans la tête du Docteur Baum qui lisait son testament (Das Testament des Dr Mabuse / Le Testament du Dr. Mabuse, 1932-33). Mais il s'agit aussi de contenter le "code Hays", système d'autocensure hollywoodien qui veillait à ne pas laisser un assassin sans châtiment à la fin d'un film, même si le film n'a pas eu trop de soucis avec la Production Code Administration, le bureau de Joseph Breen, « autocenseur » en chef ${ }^{20}$. Dans La Chienne, le meurtrier devenu clochard s'exclamait, « la vie est belle » sur les dernières images : une fin impossible à reproduire aux Etats-Unis, quatorze ans plus tard. Il est donc nécessaire d'effrayer le personnage, et avec lui le public, en soulignant que la conscience du personnage le condamne à la folie. Le sifflement de ces voix de fantômes est si insupportable à Christopher Cross qu'il tente de se suicider, ce que le code Hays ne tolérait pas davantage. Il est donc sauvé par des voisins, mais à peine est-il décroché de sa pendaison et ranimé que les voix résonnent de nouveau en over. On le retrouve ensuite passant la nuit sur un banc, houspillé par deux policemen. Ceux-ci expliquent qu'il est fou et qu'il veut être jugé et exécuté. Dans le dernier plan du film, il déambule seul et les voix continuent de se moquer de lui, d'un rire crispant.

Trois ans plus tard, Fritz Lang utilise encore la voix over dans un film où le travail « mental » du personnage principal est fondamental. Dans Le Secret derrière la porte (Secret beyond the Door, sorti en 1948), le personnage de Celia Lamphere joué par Joan Bennett cherche à élucider en pleine nuit le secret du titre. Son raisonnement, pas à pas, est donné en over. Sa voix mentale s'étonne, réfléchit, s'excite, puis s'effraie. Lang voulait utiliser pour cette voix une autre actrice de façon à insister sur l'inconscient comme "quelque chose en nous que nous ne connaissons peut-être pas »"1, mais Joan Bennett 
récupéra sa voix over après une mauvaise projection-test et des modifications demandées par Universal. Accompagnée par la musique de Mikloz Rozsa, cette voix over est, une fois de plus chez Lang, murmurée. Cette fois, ce n'est plus un susurrement sifflant mais un murmure doux et concentré. Lang établit ainsi une complicité entre le public et le personnage. Cela rend le moment suivant plus effrayant encore. Quand Celia comprend qu'elle va être tuée par son mari («This is my room!»), le spectateur est terrifié car il entend la voix comme s'il était "à l'intérieur» de la victime. Cette découverte macabre laisse place à une musique étrange qui fait perdurer le malaise. Miklos Rozsa a recopié sa partition à l'envers, l'a fait jouer, puis a fait passer la bande enregistrée dans l'autre sens pour que la musique redevienne «normale, mais sonnant de façon étrange ${ }^{22}$ ». La voix over et la musique «à l'envers » provoquent une angoisse chez le spectateur qui se projette dans le personnage.

Cette technique d'assimilation du spectateur au personnage, par l'utilisation de la voix over, entraîne une terreur encore plus forte dans les années 1960 et 1970.

\section{Over(dose) d'horreur dans les années 1960 et 1970 ?}

19 Les années 1960 et 1970 sont connues comme une période d'inflation rapide du nombre de films d'horreur produits dans de nombreux pays et d'augmentation de leurs budgets. La violence et la puissance terrifique des films s'accroissent aussi. Trois exemples permettent de saisir la façon dont la voix over est utilisée dans les films d'horreur de cette période.

En 1960, en Grande-Bretagne, un réalisateur allemand, Wolf Rilla, réalise Le Village des damnés (Village of the damned). Dans les derniers instants du film, le professeur joué par George Sanders doit parvenir à cacher ses pensées alors que les enfants extraterrestres ont le don de télépathie. On entend, en voix over, la pensée sur laquelle il se concentre pour protéger ses actions : " un mur de brique ». Cette phrase résonne et se répète, alors qu'en surimpression, on distingue le mur de brique qui se lézarde, l'horloge qui avance et les enfants qui le regardent. La terreur ne provient pas de la voix over, mais du fait que cette voix over représente un système de protection qui semble défectueux face à la puissance maléfique des petites têtes blondes extraterrestres. Le son interne subjectif s'exprime sous la forme d'une voix mentale de protection. Elle représente une tentative de maîtrise de la conscience face à un danger. Même avec le flegme et l'ironie de George Sanders, la voix over du Village des damnés reste « au premier degré » et donc effrayante.

Dans le film policier d'un nouveau genre qu'invente Mario Bava deux ans plus tard en Italie, la voix over s'adresse au spectateur mais le narrateur ironise sur le film et ses personnages. Pourtant le film n'en demeure pas moins horrible. Avec La Fille qui en savait trop (La Ragazza che sapeva troppo), en 1962, on considère que Bava crée le genre "giallo ". Ces films reprennent les thèmes de romans policiers à couverture jaune, mêlant une enquête à de l'érotisme et de l'horreur. Dans la Fille qui en savait trop, la voix du narrateur, avec un ton détaché, moqueur, presque méprisant à l'égard des personnages, semble désamorcer les moments de tension. En fait, cette voix over "documentarisante», qui décrit ce que font les touristes américaines à Rome, avec son ironie acerbe, accroît encore le suspens lors des moments les plus effrayants. Parfois, le narrateur prévient d'un danger, et anticipe sur la tension: parfois, il ne fait que décrire avec détachement et froideur un élément effrayant. Pendant l'une de ces séquences, la voix du narrateur laisse place à la voix over de l'héroïne (Nora Davis). Le narrateur reprend d'abord les 
informations qu'un personnage vient de donner («Donc, il y avait eu un crime devant cette maison.»), et pose ensuite des hypothèses (« la concierge se trompait, ou elle avait menti, ou bien...»). Il fait à la fois le travail du spectateur, qui doit penser à tous ces éléments, et celui de Nora Davis (interprétée par Leticia Roman). Après le « ou bien », la voix du narrateur se tait, et une montée chromatique rapide dans les cordes amplifie le suspens. En fait, il n'y a pas de danger réel, mais Nora fait tomber une étagère et découvre des indices concernant des meurtres perpétrés des années plus tôt. Nora lit des coupures de presse les concernant. Pendant cette lecture, sa voix en over crée une tension et s'oppose à la voix mi-ironique mi-documentaire du narrateur. Ces indices s'enchaînent pour montrer que la prochaine victime doit être Nora elle-même. «Personne n'a pris au sérieux les coups de téléphone qui annonçaient les crimes», explique la voix interne subjective de la jeune femme, avant que le téléphone ne se mette à sonner. Dans la séquence suivante, alors que deux médecins discutent avec Nora au restaurant, la voix du narrateur revient pour prévenir que seule la vérité pouvait tranquilliser la jeune femme, et " qu'elle devait chercher seule la vérité, même si elle devait risquer sa vie pour cela ». Cette fois, la voix du narrateur n'est plus ironique: au contraire, elle est essoufflée, inquiète, et transmet la peur en laissant en suspend la dernière phrase.

Ce film montre une très grande liberté face aux techniques habituelles de voix over. Tous les tons sont utilisés, mais au bout du compte, que ce soit par une ironie anempathique, ou au contraire par une attitude prévenante, le résultat est le même : la tension s'accroît. La peur est distillée par les voix over, même quand un gag ou une moquerie semblent désamorcer l'effroi. On peut imaginer un bonimenteur facétieux, au temps du muet, qui aurait pu mettre de l'ironie en commentant les films. C'est un des rares cas où nous pouvons constater une filiation possible entre la voix de commentaire sur images muettes et la voix over liée à la peur.

Enfin, au début des années 1970, les nouvelles formes de l'horreur, inspirées par le film de Zombie politique (George Romero, Night of the Living Deads / La Nuit des morts-vivants, 1968) et le développement du "gore » donnent lieu à d'étranges expériences. Un film comme Messiah of Evil (Le Messie du Mal, 1973) de Willard Huyck, oscille entre le film d'auteur avec des références picturales (peintures imitant celles de Jacques Monory) et la série $\mathrm{Z}$ de zombies dévorant la chair humaine. Même après plusieurs visions, le scénario reste assez obscur. Une narratrice explique qu'elle part à la recherche de son père, un peintre qui s'est retiré dans une petite ville côtière. Sa voix raconte en flash-back tout ce qui lui est arrivé, mais parfois, elle lit le journal intime de son père et la voix over devient alors celle du père. L'enchâssement des deux voix over pourrait signifier que la voix du père pénètre l'esprit de la fille. Or, il semble que le père soit en train de devenir lui-même un zombie cannibale. La voix over de la fille signale la compréhension progressive des événements, mais crée encore plus d'effroi devant la situation catastrophique, voire apocalyptique sur la fin. Cette voix mentale semble aussi basculer dans la folie.

\section{Conclusion}

Lorsque le «bonimenteur » de vues essaye d'effrayer le public, il peut se contenter, à la fin du XVIII ${ }^{\text {ème }}$ siècle, de faire un bruit de fantôme comme dans le cadre des spectacles de fantasmagories créés par Robertson. Mais il semble que les lanternistes aient fait beaucoup plus de commentaires pédagogiques ou comiques que de récits horribles, d'après les collections de plaques retrouvées pour l'instant ${ }^{23}$. Néanmoins, un 
commentaire décalé, détaché ou glaçant, pouvait créer une ambiance effrayante sur des images adéquates. De la même façon, le conférencier de vues cinématographiques pouvait créer un moment de panique en choisissant un ton et un vocabulaire correspondant à des images terrifiantes.

Dans les exemples que nous avons vus, on remarque que l'ambiance angoissante peut être obtenue aussi bien dans le cadre d'images d'actualités que pour des films de fiction. Ce mélange entre « commentaire documentaire » et voix posée sur une aventure effrayante se retrouve dans les films parlants. C'est justement dans ce registre intermédiaire que se situent les deux premiers exemples que nous avons trouvés. Dans The Terror, la voix est "presque over ». Le présentateur qui annonce le générique se cache, crée de l'effroi et reste une sorte de médiateur avec le public comme l'était le conférencier du muet. Ce statut de présentateur revient dans bon nombre de films du début du parlant, comme nous l'avons signalér ${ }^{24}$. La voix over du générique du Mystère de la chambre jaune veut effrayer le spectateur, ou au moins le préparer à l'angoisse, tout en insistant sur la qualité des techniciens et acteurs participant au film. On note encore une fois le rôle de médiation avec le public, prévenant de l'aspect lugubre et l'en informant de façon lyrique. Par contre, dans des films comme Rebecca, I walked with a Zombie, Gaslight, Scarlet Street ou Secret beyond the Door, la voix de la conscience ou du souvenir peut mettre mal à l'aise le spectateur, car ces films laissent une zone d'ombre. La voix over intime, parfois murmurée (chez Lang) projette le spectateur à la place du / de la protagoniste. Le doute sur la santé mentale du personnage touche le public qui a peur de basculer dans la folie avec le protagoniste. L'intimité partagée avec les pensées du personnage effraie. Grâce à la voix over, l'effroi s'insinue en nous.

Cette terreur intime devient quasi apocalyptique dans Le Village des damnés. Nous souffrons avec George Sanders qui essaye de protéger ses pensées, mais nous sommes effrayés autant par les enfants monstrueux que par le suicide final du héros. De la même façon, les voix over du Messie du mal nous incitent à vouloir protéger l'héroïne de l'apocalypse qu'elle annonce. Mais on voudrait aussi se détacher de cette voix mentale s'il s'avère que le personnage est fou. Enfin, dans La Fille qui en savait trop, la voix over jongle avec toutes les tonalités possibles et l'ironie domine. Cette narration plutôt anempathique n'empêche pas la peur, bien au contraire. Les quelques exemples que nous avons analysés montrent que la voix over est un procédé d'une grande souplesse qui permet d'insuffler la peur de façon très variée.

\section{ANNEXES}

Historien du son au cinéma, Martin Barnier a publié En Route vers le parlant (CEFAL, 2002); Des films français made in Hollywood. Les versions multiples (1929-1935) (L'Harmattan, 2004); Bruits, cris, musiques de films (Presses Universitaires de Rennes, 2010) et Analyse de film : Conte d'été de Rohmer (Vrin, 2011) ce dernier co-écrit avec Pierre Beylot. Il a également codirigé divers ouvrages dont Les Biopics du pouvoir politique de l'Antiquité au XIXe siècle. Hommes et femmes de pouvoir à l'écran, codirection avec Rémi Fontanel, Lyon, Aléas, 2010. 


\section{NOTES}

1. Sur la domination des voix masculines: Amy Lawrence, Echo and Narcissus: Women's Voices in Hollywood Cinema, Berkeley, University of California Press, 1991.

2. Jean Châteauvert distingue la voix de narration hétérodiégétique (de documentaire par exemple) du narrateur en voix over. Des Mots à l'image. La Voix over au cinéma, Québec / Paris, Nuit Blanche éditeur / Méridiens Klincksieck, 1996, p. 44.

3. Sarah Kozloff, Invisible Storytellers. Voice-over narration in American fiction films, Berkeley, University of California Press, 1988.

4. Alain Boillat met la voix-over (il l'écrit ainsi) comme «point de mire de son étude » car «la conception graduelle du désancrage de la voix par rapport à l'image culmine dans ce procédé ", in Du Bonimenteur à la voix-over. Voix-attraction et voix-narration au cinéma, Lausanne, Antipodes, 2007, p. 30.

5. Georges Dalbe, Conférencier au Nouveau Cinéma, lettre publiée dans Le Courrier cinématographique, Paris, 14 octobre 1911. Repris in Iris, n² 22, Automne, 1996, pp. 31-32.

6. Martin Barnier, Bruits, cris, musiques de films. Les projections avant 1914, Rennes, PUR, 2010, pp. 27-33.

André Gaudreault, Cinéma et attraction. Pour une nouvelle histoire du cinématographe, Paris, CNRS, 2008. On utilisait, entre 1980 et 2008 l'expression « cinéma des premiers temps ».

7. Article du Courrier des spectacles, n 1086, 23 février 1800, p. 4 cité par Laurent Mannoni, Le Grand art de la lumière et de l'ombre. Archéologie du cinéma, Paris, Nathan, 1994, p. 156.

8. Un enregistrement d'explications pédagogiques d'un film peut être entendu sur Jérusalem, film restaurédans le DVD joint au livre dirigé par Giusy Pisano et Valérie Pozner, Le Muet a la parole. Cinéma et performance à l'aube du XXe siècle, Paris, AFRHC, 2005.

9. Aujourd'hui avenue Jean-Médecin.

10. L'Éclaireur de Nice, 31 mars 1907.

11. Tract publicitaire écrit par Louis Feuillade en novembre 1915, cité in Francis Lacassin, Louis Feuillade, maître des lions et des vampires, Paris, Bordas, 1995, cité in Plaquette du DVD Gaumont, Les Vampires, (2006), p. 3.

12. Ce terme est important pour comprendre la transformation progressive qui eut lieu dans le cinéma. Cf. Martin Barnier, En route vers le parlant. Histoire d'une évolution technologique, économique et esthétique du cinéma (1926-1934), Liège, Éditions du CÉFAL, 2002.

13. Donald Crafton, The Talkies, New York, Charles Scribner's Sons, 1997, p. 549.

14. Jean-Pierre Coursodon, La Warner Bros., Paris, Centre Pompidou, 1991, p. 85.

15. Alain Boillat commente cela, op. cit., pp. 302-313.

16. Sarah Kozloff, op. cit., pp. 45-47.

17. Ibid., p. 108.

18. Michel Chion, Un art sonore, le cinéma, Paris, Cahiers du cinéma, 2003, p. 413.

19. Ibid., p. 425.

20. Bernard Eisenschitz, Fritz Lang au travail, Paris, éditions Cahiers du cinéma, 2011, p. 175.

21. Lang cité par Eisenschitz, ibid., p. 184.

22. Ibid., p. 183.

23. Jacques Perriault, Mémoires de l'ombre et du son. Une archéologie de l'audio-visuel, Paris, Flammarion, 1981.

24. Martin Barnier, «La voix qui présente », Cinémas, Université de Montréal, vol. 20, $\mathrm{n}^{\circ} 1$, automne 2009, pp. 91-111. 


\section{RÉSUMÉS}

Les voix over de narration peuvent servir à effrayer les spectateurs de cinéma. Depuis les bonimenteurs-conférenciers des salles d'avant 1914, jusqu'aux films d'horreur des années 1970, en passant par les films des années 1930 et 1940, les voix placées sur des images peuvent créer une atmosphère lugubre. Le contraste entre une voix susurrée et des images pleines de suspens, est générateur d'angoisse. Des exemples tirés de films de Lang, Hitchcock, Tourneur, ou de cinéastes bien moins connus, permettent de vérifier que les voix over s'intègrent dans une longue tradition mais qu'elles évoluent constamment.

Voice over can be use to frighten movie audiences. From the film lecturer before 1914 to the 1970s Horror movies, voices accompanying images can create a gloomy atmosphere. Contrast between suspenseful images and a whispering voice can generate fear. With examples taken from Lang, Hitchcock, Tourneur, and unknown directors, we will show that voice over creates fear since a long tradition. But each time it evolves in a different way.

\section{INDEX}

Index chronologique : XXème siècle

Mots-clés : angoisse, effroi, films, histoire du cinéma, Hitchcock, Lang, peur, son, Tourneur, voix over

Index géographique : Europe et USA

\section{AUTEUR}

\section{MARTIN BARNIER}

Professeur en études cinématographiques et audiovisuelles à l'Université Lumière Lyon 2, Equipe d'Accueil PASSAGES XX-XXI (EA4160) 